\title{
Expression analysis of intracellular vesicle trafficking superfamily genes, CaRab-GTP, in response to drought, dehydration and salinity in leaves of chickpea (Cicer arietinum L.)
}

\author{
Khassanova G. ${ }^{1 *}$, Jatayev S. ${ }^{1}$, Kurishbayev A. ${ }^{1}$, Langridge P. ${ }^{2,3}$, Schramm C. ${ }^{4}$, \\ Jenkins C. ${ }^{4}$, Soole K. ${ }^{4}$, Shavrukov Y. ${ }^{4}$ \\ ${ }^{1}$ Faculty of Agronomy, S. Seifullin Kazakh AgroTechnical University, Nur-Sultan, Kazakhstan \\ ${ }^{2}$ University of Adelaide, SA, Australia \\ ${ }^{3}$ Wheat Initiative, Julius Kühn-Institute, Berlin, Germany \\ ${ }^{4}$ College of Science and Engineering, Biological Sciences, Flinders University, Australia \\ *e-mail: khasanova-gulmira@mail.ru
}

Introduction and Aim: Environmental stresses such as drought and salinity inhibit plant growth and productivity. Chickpea is an important legume, moderately tolerant to high temperatures, drought and salinity stress during the growing season. CaRab-GTP, intracellular vesicle trafficking superfamily genes, play essential role in response to these stresses. $C a R a b C$, belonging to the family of Rab-GTP genes, was identified from an SNP database using bioinformatic and molecular genetic analyses. The aim of this study was to identify and analyse the role of $C a R a b C$ in tolerance to drought, salinity and rapid dehydration in chickpea.

Methods: Bioinformatics and systems biology methods were applied in this study to confirm the potentially important role of the target gene in tolerance to abiotic stresses in chickpea. Three experiments applying abiotic stress treatments (salinity, slow drought and rapid dehydration) were carried out. For gene expression, RNA was extracted from control and stressed plants with subsequent cDNA synthesis and qPCR analysis. For SNP identification, Amplifluor SNP analysis, sequencing and bioinformatics were used. Results: Eight sub-families with 54 isoforms of CaRab genes were identified and clearly distinguished in the phylogenetic tree based on protein sequences. Levels of CaRabC expression were very high in plants subjected to salinity and rapid dehydration, but down-regulated under slowly developing drought. Five isoforms of CaRabC were strongly stress- and genotype-dependent, showing differential expressions.

Conclusion: CaRabC is part of the large Rab-GTP gene family. All five isoforms were expressed differently in response to salinity, rapid dehydration and drought. This confirms the important role of this gene in the tolerance of chickpea plants to abiotic stresses. Acknowledgements: This study was supported by the Ministry of Education and Science. Kazakhstan, Research program BR05236500 (SJ). 